partial derivative of the line flux with respect to the continuum flux, averaged over each paraboloidal surface of constant delay). Unfortunately this requires many observations to describe adequately the light curves, and the interval between observations must be chosen carefully to match the relevant timescales.

Such careful sampling is difficult to achieve in ground-based astronomy because observing is subject to uncontrollable interruptions, of which obscuration by the Moon's light and by bad weather are the worst examples. But orbiting satellites are subject to neither of these constraints, and so in 1987 a campaign was organized to use the International Ultraviolet Explorer satellite to observe the Seyfert galaxy NGC5548 every four days over a span of eight months. From this collection of spectra, time series for three continuum bands and eight emission lines were compiled. At the same time, a large-scale complementary ground-based campaign was mounted to gather data (albeit with less regular sampling) on the behaviour of the optical continuum and emission lines.

\section{Ionization conditions}

With these data in hand it has become feasible to attempt the deconvolution. The result is that (at least in this example) the lines fall neatly into two groups: those radiated in relatively high-ionization conditions ( $\mathrm{H}$ II, N v, C IV, Si IV and Lyman $\alpha$ ) and those from lower-ionization conditions ( $\mathrm{C}_{\mathrm{III}}$ ], $\mathrm{Mg}$ II, $\mathrm{O}_{\mathrm{I}}$ and $\mathrm{H} \beta$ ). The first set responds most strongly to changes in the continuum at zero lag, the response tapering off almost to zero after 20 days. The second set responds after a considerably greater delay, but only for the $\mathrm{C}_{\text {III] }}$ and $\mathrm{H} \beta$ (ref. 7) lines was the signal-to-noise ratio high enough to permit a good look at the response function: for both of these lines, the response at zero delay is quite weak, builds to a peak after 20 to 30 days, and falls steeply at larger lags.

Such one-dimensional maps are interesting, but they are less than satisfactory because they give the marginal emissivity only as a function of delay along our line of sight. Whatever symmetry truly characterizes the emission-line region, it is very unlikely to be defined by our line of sight. The shapes of the response functions, however, give a good guide to the true symmetry. When the lines are radiated isotropically (a good approximation for many of those measured), response functions peaking at zero lag must be associated with quasi-spherical regions, whereas the simplest symmetry for those peaking at larger lags is cylindrical. Thus it seems that (at least in NGC5548) the emission-line region has two sub-regions: an inner, roughly spherical zone responsible for the high-ionization lines and an outer, flattened zone responsible for the low-ionization lines.

The fact that five response functions are obtained from the same (high-ionization) region permits a still more detailed analysis. At each lag $\tau$, the overall amplitude of the high-ionization response functions reveals the solid angle subtended by the highly ionized material on a sphere of diameter $c \tau$; the ratios of these functions reveal (with the help of some atomic-physics calculations) the physical conditions in the gas. Adding into the analysis the mean ionizing power, a new estimate may be made of the distance from the continuum to the line-emitting region; satisfyingly, this distance corresponds to the radius of the sphere $(c \tau / 2)$ to within a factor of 2-4. Such selfconsistency adds considerable confidence to the inferences drawn about physical conditions: the high-ionization lineemitting material in NGC5548 is spread over spherical shells ranging in radius from 4 to 14 light-days, with the greatest amount of line radiation coming from the shell with a radius of about 8 light-days; the pressure stays fairly constant throughout this region, maintaining a density of around $10^{11} \mathrm{H}$ atoms $\mathrm{cm}^{-3}$; and the thickness of individual line-emitting clumps is less than about $10^{11} \mathrm{~cm}$.

Because only two lines are available to constrain the models, the structure of the outer (low-ionization) region is determined less well. Although it must be flattened cylindrically, the response functions could correspond equally well to a ring with a radius of about 25 light-days lying face-on to our line of sight, or a ring with a radius of about 100 light-days viewed almost edge-on.

These studies are producing a higher level of understanding of active-galaxy emission lines. Both direct confirmation of inferences from models and detailed structural information are now accessible, provided that the necessary large-scale coordinated efforts can be mounted. Judging by the number of new monitoring programmes being promoted at the meeting, that is a cost that will soon be met.

Julian H. Krolik is in the Department of Physics and Astronomy, Johns Hopkins University, Baltimore, Maryland 21218, USA.

1. Clavel, J. et al. Astrophys. J. (in the press).

2. Clavel, J. in Variability in Active Galactic Nuclei (eds Miller H.R. \& Wiita, P.J.) (Cambridge University Press, in the press)

3. Peterson, B. in Variability in Active Galactic Nuclei (eds Miller, H.R. \& Wiita, P.J.) (Cambridge University Press, in the press).

4. Krolik, J.H. in Variability in Active Galactic Nuclei (eds Miller, H.R. \& Wiita, P.J.) (Cambridge University Press, in the press).

5. Bahcall, J.N., Kozlovsky, B.-Z. \& Salpeter, E.E. Astrophys 171, 467 (1972).

6. Blandford, R.D. \& McKee, C.F. Astrophys. J. 255, 419 1982

7. Horne, K. in Variability in Active Galactic Nuclei (eds Miller, H.R. \& Wiita, P.J.) (Cambridge University Press, in the press).

\section{Equilibrated junk}

WHAT should we do with our rubbish? Dumping it as landfill is increasingly unacceptable, clear-air lobbyists oppose incineration even at sea, and few citizens are dedicated enough to sort and classify the stuff for recycling. Daedalus recalls composting, that ancient method of reducing organic waste to a uniform and fertilizing humus. He is now adapting the technique to urban rubbish.

His idea is simply to heat the rubbish to such a temperature that all possible chemical reactions between its components go to completion. The end product, DREADCO's 'Rumus', would be the stablest, lowest-energy compound or mixture of compounds that could be formed from the initial composition. The concept will be familiar to those astrochemists who calculate what minerals would preferentially separate from any assumed primaeval planetary material as it cooled. Rubbish presents a harder challenge. The only safe bets are that Rumus will be extensively chemically bonded, and will therefore be a solid; and since it has the lowest energy possible for its composition, its formation will give out heat.

So while DREADCO's chemists wrestle with thermodynamic equilibria in rubbish, its chemical engineers are making Rumus in practice. They are compacting carefully defined mixtures of plastic bags and bottles, old newspapers, tin cans, broken china, disposable nappies and takeawaymeal residues at high temperatures and pressures, measuring the heat evolved and studying the product. From the results, they hope to design a pilot plant to carry out this self-heating reaction automatically and continuously.

Raw Rumus will be an uninspiring, brownish solid. At first Daedalus hoped that it might be hard and strong enough for road-stone or breeze blocks. But he then recalled that a mixture always has a lower melting point than its ingredients. Rumus will be such a horrendous mixture that its melting point will be quite low. Inert, softish and yielding, it will be ideal for asphalt, roof sealants, caulking and the like. It may, of course, contain two or more intermixed phases of different melting point, thus being self-reinforced into a tough, useful solid.

But to command a wide and varied market, it will probably need a bit of modification. The incoming rubbish may have to be spiked with conditioners like sulphur, to vulcanize and toughen the organic components; or silicon, which should combine with metals, organics and inorganics, to merge otherwise immiscible phases. Once the process has been optimized, DREADCO's Rumus will solve the whole refuse disposal problem, and make a splendid profit into the bargain.

David Jones 